\title{
A Collection of 2D Elliptic Problems for Testing Adaptive Grid Refinement Algorithms 米
}

\author{
William F. Mitchell ${ }^{\mathrm{a}}$ \\ ${ }^{a}$ National Institute of Standards and Technology, 100 Bureau Dr., Stop 8910, Gaithersburg, MD, \\ 20899-8910
}

\begin{abstract}
Adaptive grid refinement is a critical component of the improvements that have recently been made in algorithms for the numerical solution of partial differential equations (PDEs). The development of new algorithms and computer codes for the solution of PDEs usually involves the use of proof-of-concept test problems. 2D elliptic problems are often used as the first test bed for new algorithms and codes. This paper contains a set of twelve parametrized 2D elliptic test problems for adaptive grid refinement algorithms and codes. The problems exhibit a variety of types of singularities, near singularities, and other difficulties.
\end{abstract}

Keywords: adaptive grid refinement, standard test problems, benchmarking 2010 MSC: 65N50, 65N30

\section{Introduction}

The numerical solution of partial differential equations (PDEs) is the most computeintensive part of a wide range of scientific and engineering applications. Consequently the development and application of faster and more accurate methods for solving partial differential equations has received much attention in the past fifty years. Self-adaptive methods to determine a quasi-optimal grid are a critical component of the improvements. Although adaptive grid refinement techniques are now in widespread use in applications, they remain an active field of research, particularly in the context of $h p$-adaptive techniques.

The development of new algorithms and computer codes for the solution of PDEs usually involves the use of proof-of-concept test problems. Such test problems have a variety of uses such as demonstrating that a new algorithm is effective, verifying that a new code is correct in the sense of achieving the theoretical order of convergence, and comparing the performance of different algorithms and codes. Nearly every paper on

\footnotetext{
Contribution of NIST, not subject to copyright.

Preprint. Appeared in Appl. Math. Comput., 220, 2013, pp. 350-364.

Email address: william.mitchell@nist.gov (William F. Mitchell)

$U R L:$ http://math.nist.gov/ WMitchell (William F. Mitchell)
} 
algorithms for solving PDEs contains a numerical results section with one or more test problems.

In this paper, the focus is on 2D elliptic problems, as they are often used as the first test bed for new algorithms and codes for solving PDEs. Other classes of problems (3D, hyperbolic, goal-oriented, etc.) can be found on the NIST Adapative Mesh Refinement Benchmark Problems web page [1. This paper contains a set of twelve 2D elliptic test problems for adaptive grid refinement algorithms and codes. Most of the problems are taken from the numerical results section of papers in the adaptive grid refinement literature. The problems exhibit a variety of types of singularities (point and line singularities on the boundary and in the interior), near singularities (sharp peaks, boundary layers, and wave fronts), and other difficulties. Most of the problems are parametrized to allow "easy" and "hard" variations on the problem. This collection of problems can be used for testing correctness of a computer code or optimality of an algorithm by observing the convergence rate of a norm (energy, $H^{1}, L^{2}$, etc.) of the error with respect to the number of degrees of freedom, to test the efficiency index of error estimates, to benchmark the computation time of different computer codes and algorithms, etc.

We primarily consider elliptic partial differential equations of the form

$$
\begin{aligned}
-\frac{\partial}{\partial x}\left(p(x, y) \frac{\partial u}{\partial x}\right)-\frac{\partial}{\partial y}\left(q(x, y) \frac{\partial u}{\partial y}\right)+r(x, y) u & =f(x, y) \text { in } \Omega \\
u & =g_{D}(x, y) \text { on } \partial \Omega_{D} \\
p(x, y) \frac{\partial u}{\partial x} \frac{\partial y}{\partial s}-q(x, y) \frac{\partial u}{\partial y} \frac{\partial x}{\partial s}+c(x, y) u & =g_{N}(x, y) \text { on } \partial \Omega_{N}
\end{aligned}
$$

with coefficient functions $p, q, r$ and $c$, and right hand sides $f, g_{D}$ and $g_{N}$, where $\Omega$ is a bounded, connected, polygonal, open region in $\mathbb{R}^{2}$ with boundary $\partial \Omega=\partial \Omega_{D} \cup \partial \Omega_{N}$, $\partial \Omega_{D} \cap \partial \Omega_{N}=\emptyset$. Differentiation with respect to $s$ is with respect to a counterclockwise parametrization of the boundary $(x(s), y(s))$ with $\|(d x / d s d y / d s)\|=1$. Equation 2 represents Dirichlet boundary conditions, and Equation 3 represents natural boundary conditions if $c=0$ and mixed boundary conditions otherwise. We assume the data in Equations 1 1 3 satisfy the usual ellipticity and regularity assumptions. Some of the test problems extend this to a coupled system of two equations, and the inclusion of first order derivative and mixed derivative terms.

Many of the test problems use the special case of Poisson's Equation

$$
-\nabla^{2} u:=-\frac{\partial^{2} u}{\partial x^{2}}-\frac{\partial^{2} u}{\partial y^{2}}=f(x, y)
$$

and Laplace's Equation, which is Poisson's equation with $\mathrm{f}=0$. All but one problem are defined by the method of manufactured solution. When the right side of the equation is simply given as " $f$ " and the exact solution is known, $f$ is determined by applying the operator to the exact solution. Similarly, if the boundary conditions are said to be Dirichlet and the exact solution is known, $g_{D}$ is given by the exact solution.

We use the terms singular and singularity rather loosely. We consider a function to be singular (or to have a singularity) if it or a derivative of any order is singular. More precisely, we consider it to be singular if there exists a finite positive $m$ such that the function does not lie in $H^{m}$, where $H^{m}$ is the usual Sobolev space of functions whose 

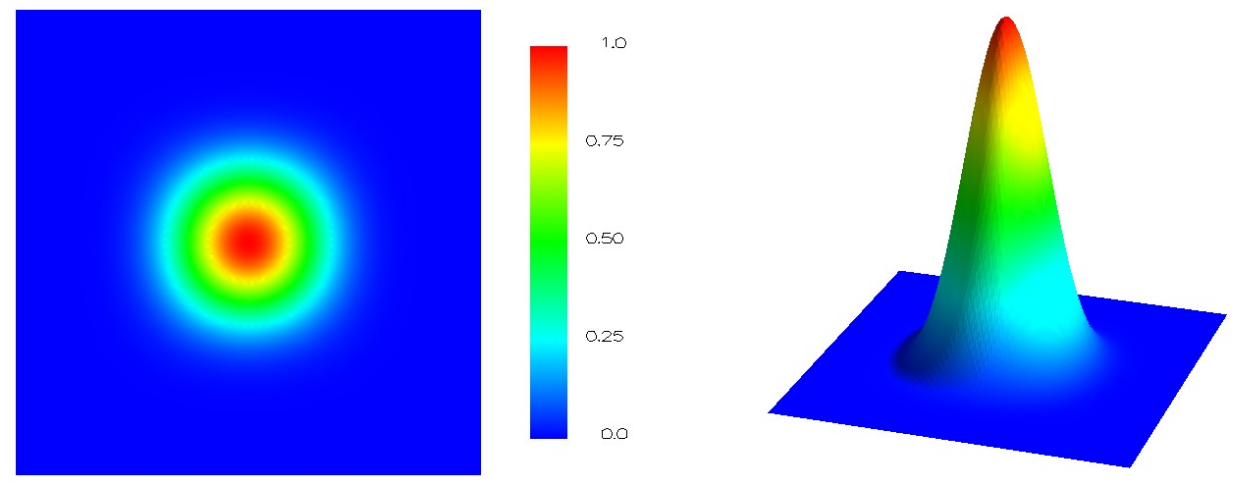

Figure 1: The solution of the analytic problem with $a=10$.

derivatives of order $m$ are square integrable, and the usual extensions to noninteger $m$. We refer to the largest $m$ such that the function is in $H^{m}$ as the Sobolev regularity of the function.

\section{The Set of Test Problems}

\subsection{Analytic Solution}

This is a well behaved problem with a smooth solution that has no trouble spots. It can be used for seeing how an adaptive algorithm behaves in a context where adaptivity isn't really needed.

Equation: Poisson

Domain: unit square

Boundary conditions: Dirichlet

Solution: $2^{4 a} x^{a}(1-x)^{a} y^{a}(1-y)^{a}$

Parameters: the integer $a$ determines the degree of the polynomial solution. It should be chosen to be large enough that the highest order finite elements to be used will not give the exact solution.

The solution with $a=10$ is shown in Figure 1, both as a color-mapped image and as a surface in perspective. The other figures that show solutions also present these two views.

\subsection{Reentrant Corner}

For elliptic partial differential equations, reentrant corners in the domain are a source of singularities in the solution. In particular, for a corner with an angle $\omega$ as shown in Figure 2, the solution behaves like $r^{\alpha}$ where $r$ is the distance from the corner and $\alpha=\pi / \omega$. The solution is in $H^{1+\alpha-\epsilon} \forall \epsilon>0$ [2].

Equation: Laplace

Domain: $(-1,1) \times(-1,1)$ with a section removed from the clockwise side of the positive $x$ axis, as shown in Figure 2,

Boundary conditions: Dirichlet 


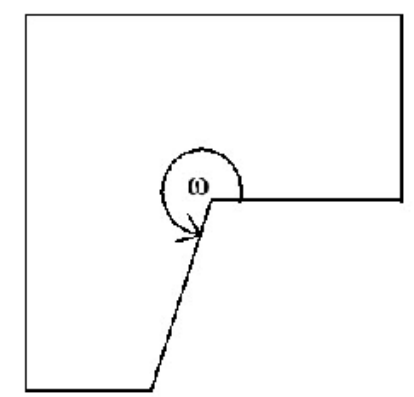

Figure 2: Domain for the reentrant corner problem.
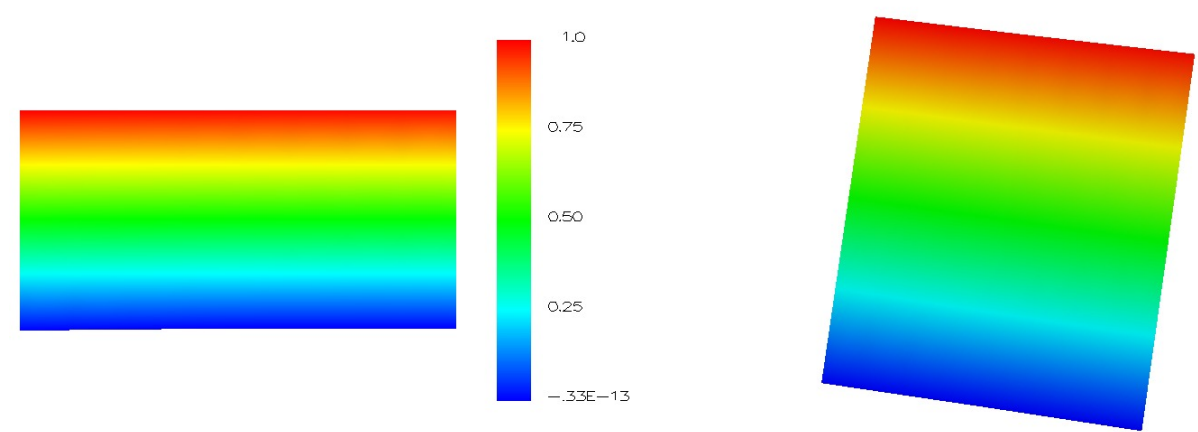

Figure 3: The solution of the reentrant corner problem with $\omega=\pi+.01$.

Solution: $r^{\alpha} \sin (\alpha \theta)$ where $r=\sqrt{x^{2}+y^{2}}$ and $\theta=\tan ^{-1}(y / x)$

Parameters: $\omega$ determines the angle of the reentrant corner, and consequently $\alpha$ and the strength of the singularity. Varying $\omega$ can be used to study the effect of the strength of the singularity on adaptive algorithms. Using $\omega=3 \pi / 2$ gives the infamous "L domain" problem used heavily in the adaptive refinement community. With $\omega=2 \pi$ the domain is a square with a slit. A solution with $\omega$ slightly larger than $\pi$ is nearly linear.

The solutions for $\omega=\pi+0.01,5 \pi / 4,3 \pi / 2,7 \pi / 4$, and $2 \pi$ are shown in Figures 3.7.

\subsection{Linear Elasticity}

Several papers [3, 4, 5, 6] use a problem from linear elasticity as an example. This is a coupled system of two equations with a mixed derivative in the coupling term. In 3 the equations are given as

$$
\left\{\begin{array}{c}
-E \frac{1-\nu^{2}}{1-2 \nu} \frac{\partial^{2} u}{\partial x^{2}}-E \frac{1-\nu^{2}}{2-2 \nu} \frac{\partial^{2} u}{\partial y^{2}}-E \frac{1-\nu^{2}}{(1-2 \nu)(2-2 \nu)} \frac{\partial^{2} v}{\partial x \partial y}=F_{x} \\
-E \frac{1-\nu^{2}}{2-2 \nu} \frac{\partial^{2} v}{\partial x^{2}}-E \frac{1-\nu^{2}}{1-2 \nu} \frac{\partial^{2} v}{\partial y^{2}}-E \frac{1-\nu^{2}}{(1-2 \nu)(2-2 \nu)} \frac{\partial^{2} u}{\partial x \partial y}=F_{y}
\end{array}\right.
$$



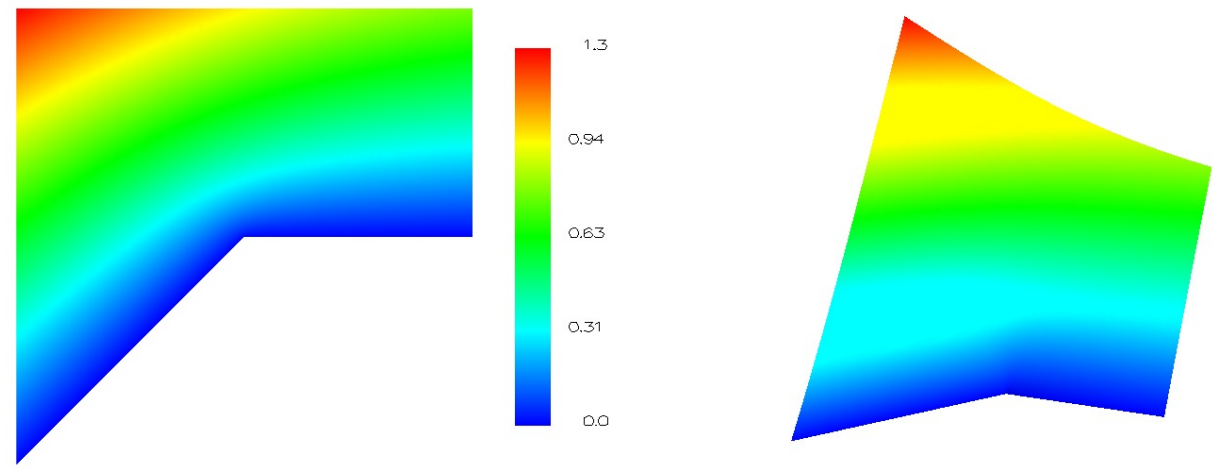

Figure 4: The solution of the reentrant corner problem with $\omega=5 \pi / 4$.
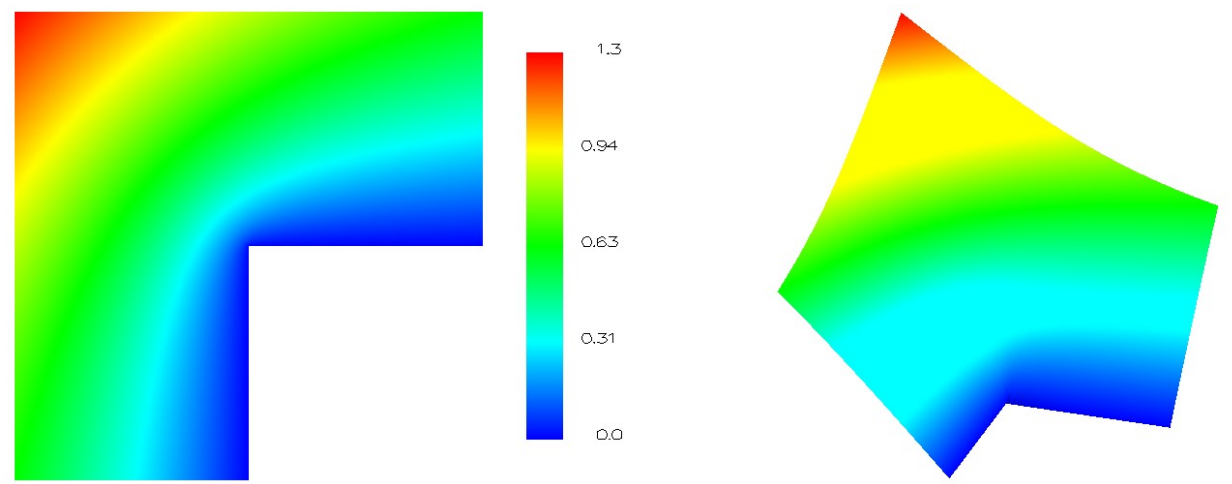

Figure 5: The solution of the reentrant corner problem with $\omega=3 \pi / 2$.
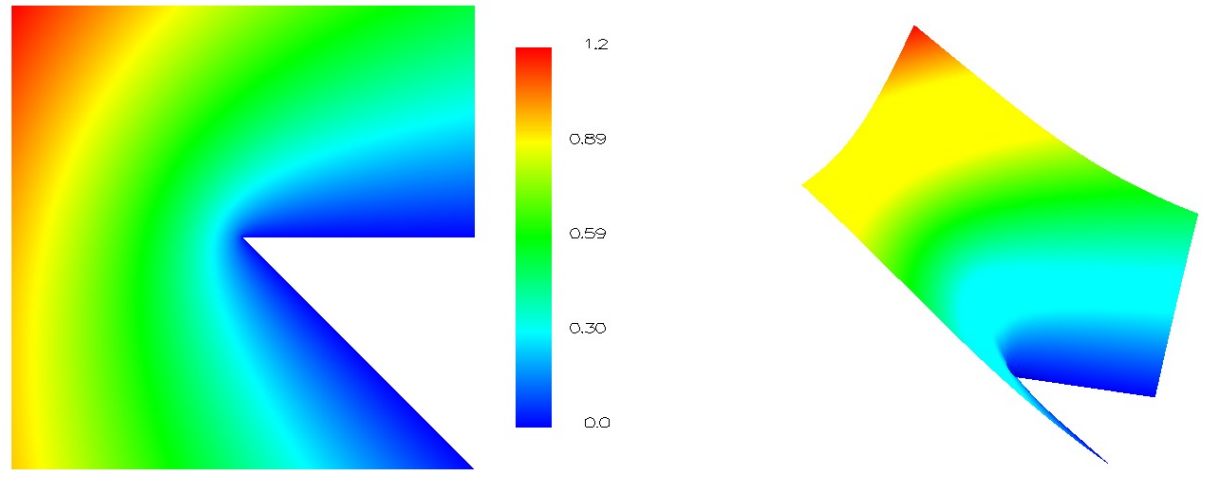

Figure 6: The solution of the reentrant corner problem with $\omega=7 \pi / 4$. 

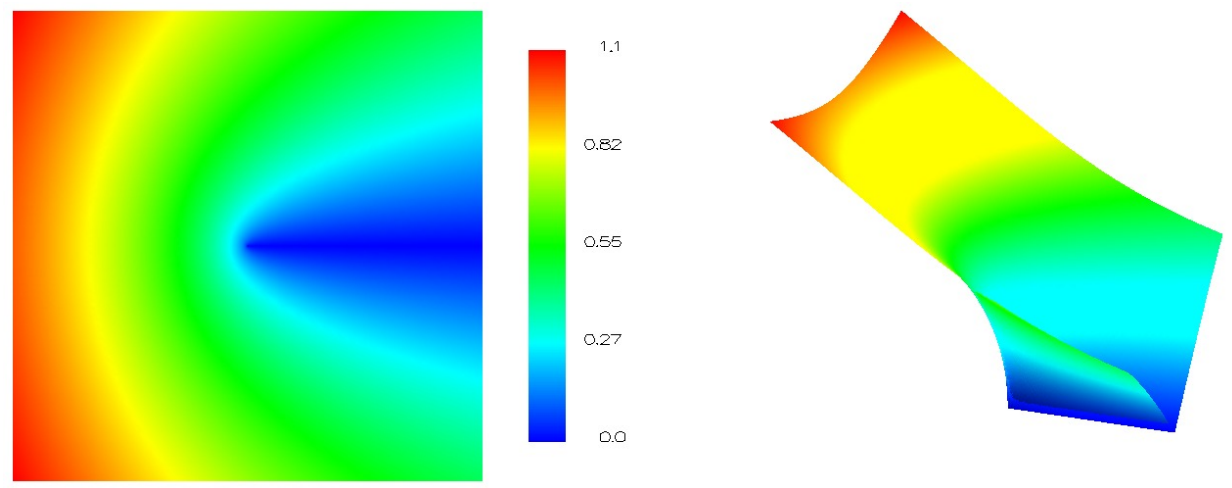

Figure 7: The solution of the reentrant corner problem with $\omega=2 \pi$.

\begin{tabular}{|c|c|c|}
\hline mode & $\lambda$ & $Q$ \\
\hline \hline 1 & 0.5444837367825 & 0.5430755788367 \\
2 & 0.9085291898461 & -0.2189232362488 \\
\hline
\end{tabular}

Table 1: Parameters for the linear elasticity problem.

where $u$ and $v$ are the $x$ and $y$ displacements, $E$ is Young's Modulus, and $\nu$ is Poisson's ratio.

Two solutions are given in 3 in polar coordinates; a mode 1 solution

$$
\left\{\begin{array}{l}
u=\frac{1}{2 G} r^{\lambda}[(\kappa-Q(\lambda+1)) \cos (\lambda \theta)-\lambda \cos ((\lambda-2) \theta)] \\
v=\frac{1}{2 G} r^{\lambda}[(\kappa+Q(\lambda+1)) \sin (\lambda \theta)+\lambda \sin ((\lambda-2) \theta)]
\end{array}\right.
$$

and a mode 2 solution

$$
\left\{\begin{array}{l}
u=\frac{1}{2 G} r^{\lambda}[(\kappa-Q(\lambda+1)) \sin (\lambda \theta)-\lambda \sin ((\lambda-2) \theta)] \\
v=-\frac{1}{2 G} r^{\lambda}[(\kappa+Q(\lambda+1)) \cos (\lambda \theta)+\lambda \cos ((\lambda-2) \theta)]
\end{array}\right.
$$

where $\kappa=3-4 \nu, G=E /(2(1+\nu))$, and $\lambda$ and $Q$ are constants. The solutions have a point singularity at the origin and are in $H^{1+\lambda-\epsilon} \forall \epsilon>0$ [4].

The domain is taken to be a square with a slit (a cracked plate) as in [6]. Some of the other references use an L-shaped domain.

Equation: coupled system of two equations given by Equation 4

Domain: $(-1,1) \times(-1,1)$ with a slit from $(0,0)$ to $(1,0)$

Boundary conditions: Dirichlet

Solution: Two solutions as given in Equations 5 and 6 .

Parameters: All the above references use $\nu=0.3$ and $E=1$. The values for $\lambda$ and $Q$ differ in the two solutions, and are given in Table 1. See [3] for the derivation of these constants. With these solutions and parameters, $F_{x}=F_{y}=0$.

The solutions are shown in Figures 8.11. 

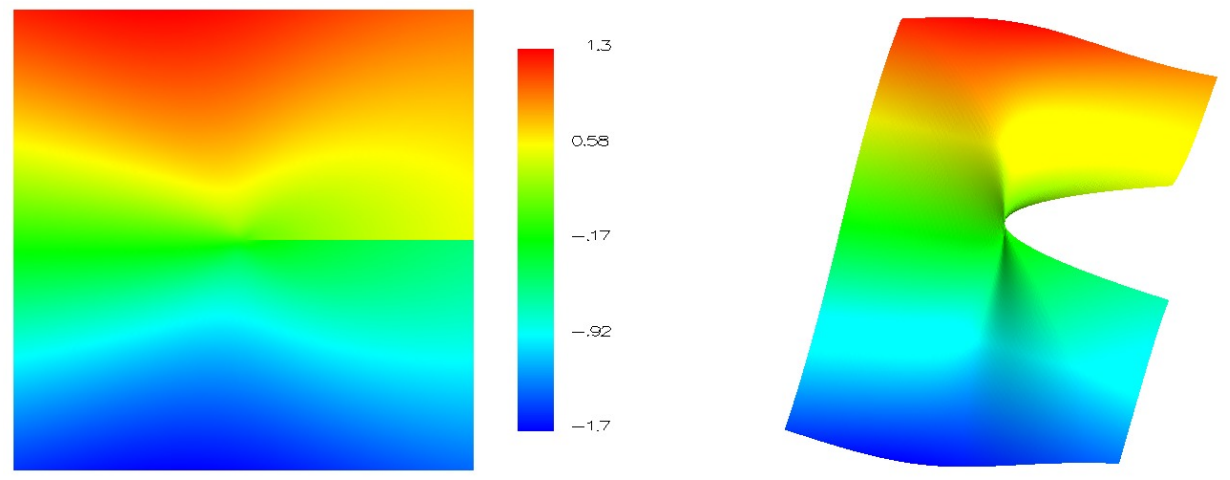

Figure 8: The $u$ component of the mode 1 solution of the linear elasticity problem.
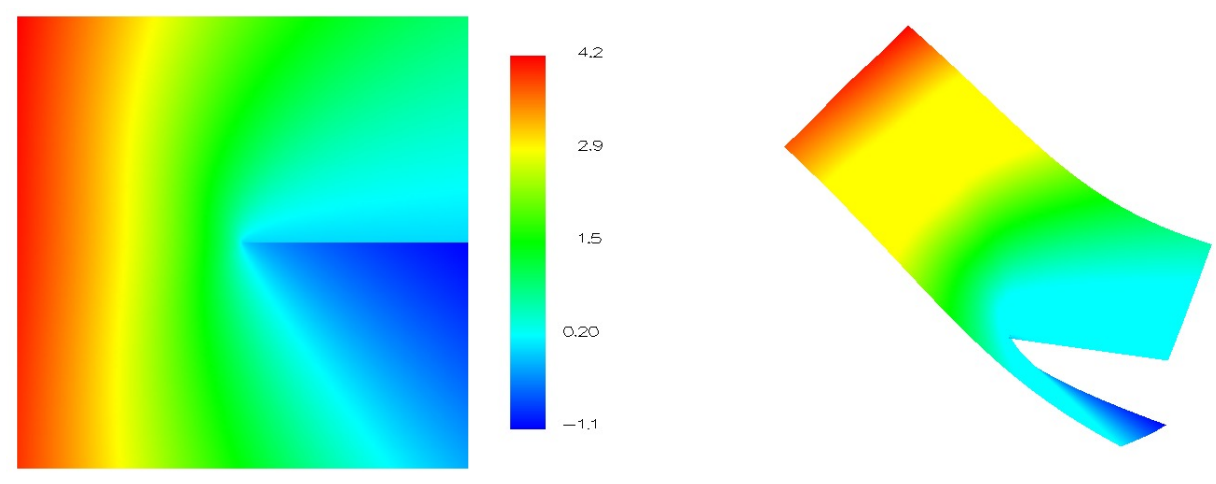

Figure 9: The $v$ component of the mode 1 solution of the linear elasticity problem.
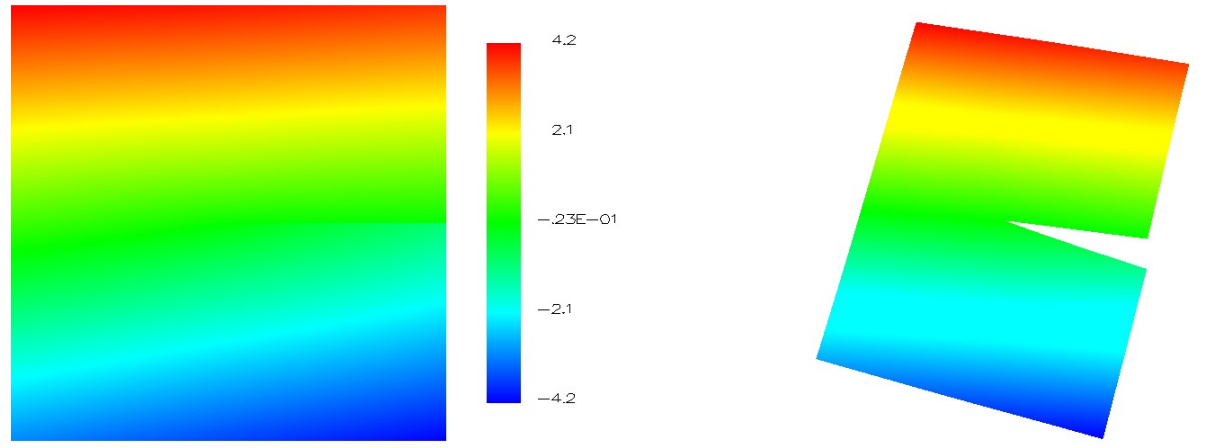

Figure 10: The $u$ component of the mode 2 solution of the linear elasticity problem. 

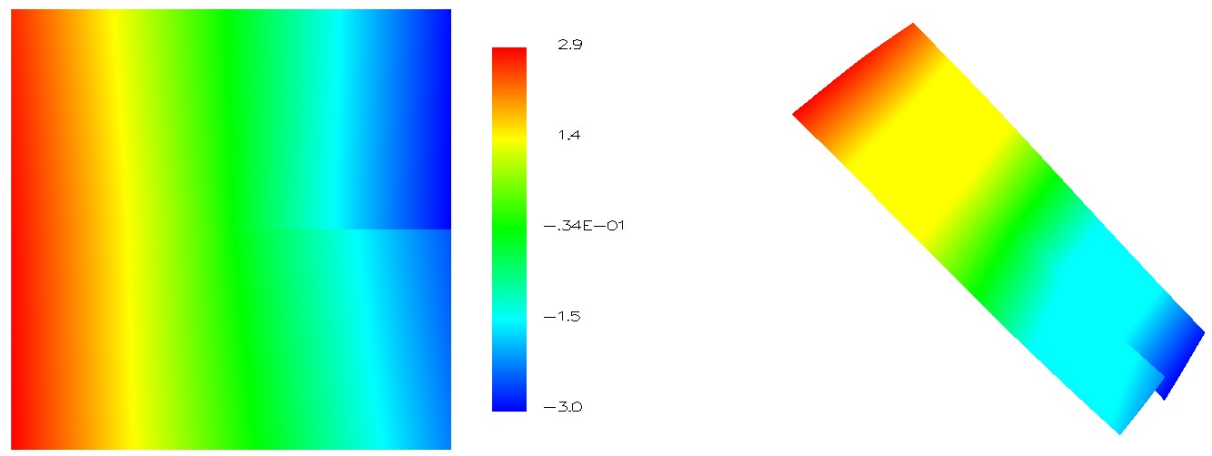

Figure 11: The $v$ component of the mode 2 solution of the linear elasticity problem.
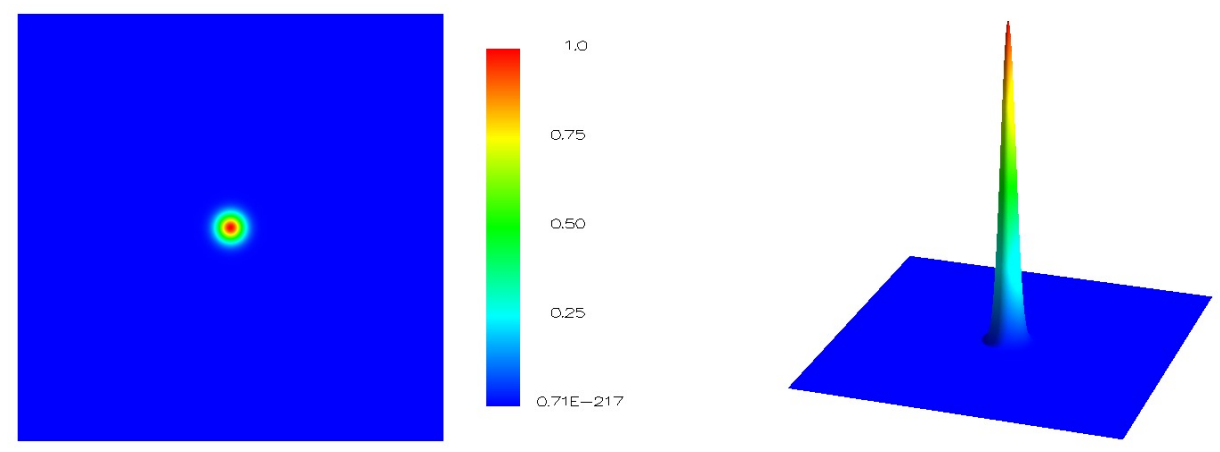

Figure 12: The solution of the peak problem with $\alpha=1000,\left(x_{c}, y_{c}\right)=(0.5,0.5)$.

\subsection{Peak}

This problem has an exponential peak in the interior of the domain. It is based on Problem 10 in [7].

Equation: Poisson

Domain: Unit square.

Boundary conditions: Dirichlet

Solution: $e^{-\alpha\left(\left(x-x_{c}\right)^{2}+\left(y-y_{c}\right)^{2}\right)}$

Parameters: $\left(x_{c}, y_{c}\right)$ is the location of the peak, and $\alpha$ determines the strength of the peak.

The solutions of two instances of this problem are shown in Figures 12 and 13 . The first has a mild peak at the vertex $(0.5,0.5)$ with $\alpha=1000$. The second has a sharp peak at $(0.51,0.117)$ with $\alpha=100000$.

\subsection{Battery}

This problem comes from [6], where it is attributed to Ivo Babuška and Sandia National Laboratories. It features piecewise constant coefficient functions $p$ and $q$ and right 

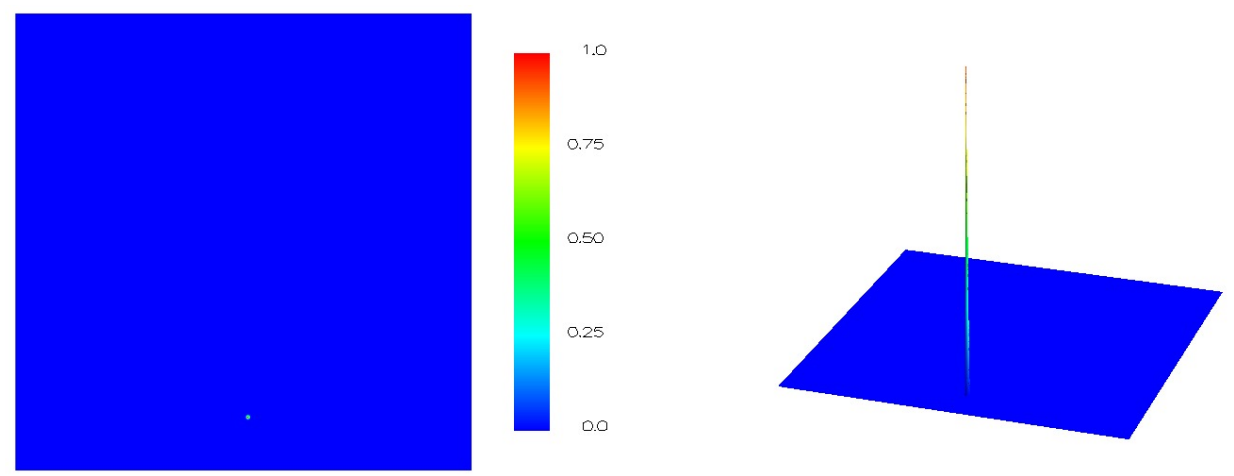

Figure 13: The solution of the peak problem with $\alpha=100000,\left(x_{c}, y_{c}\right)=(0.51,0.117)$.

\begin{tabular}{|c|c|c|c|}
\hline $\mathrm{k}$ & $p$ & $q$ & $f$ \\
\hline \hline 1 & 25.0 & 25.0 & 0.0 \\
2 & 7.0 & 0.8 & 1.0 \\
3 & 5.0 & 0.0001 & 1.0 \\
4 & 0.2 & 0.2 & 0.0 \\
5 & 0.05 & 0.05 & 0.0 \\
\hline
\end{tabular}

Table 2: Piecewise constant coefficient functions for the battery problem. $\mathrm{k}$ designates the region from Figure 14

hand side $f$, and mixed boundary conditions. The solution has multiple point singularities in the interior of the domain. The equation models heat conduction in a battery with nonhomogeneous materials. The domain is the rectangle shown in Figure 14. The numbered regions indicate the areas of different material constants, with the constants given in Table 2. The location of the line segments that separate the regions are given in Table 3. The coefficients of the mixed boundary conditions are given in Table 4 . The solution has singularities at the points where three or more materials meet. For any $\epsilon>0$ there exists coefficients such that the solution is in $H^{1+\epsilon}$. By observing the rate of convergence with uniform $h$-refinement and comparing it with the theoretical a priori error bound, we estimate that $\epsilon$ is about $1 / 2$ for the given set of coefficients. The solution is shown in Figure 15 .

Equation: $-\frac{\partial}{\partial x}\left(p \frac{\partial u}{\partial x}\right)-\frac{\partial}{\partial y}\left(q \frac{\partial u}{\partial y}\right)=f$ with $p, q$ and $f$ given in Table 2

Domain: $(0,8.4) \times(0,24)$

Boundary conditions: Mixed, with $c$ and $g_{N}$ given in Table 4

Solution: Unknown.

Parameters: None.

\subsection{Boundary Layer}

This problem comes from [8]. It has an $O(\epsilon)$ boundary layer along the right and top sides of the domain. It is a convection-diffusion equation with first order terms. 


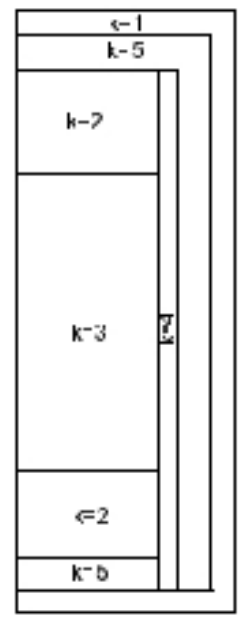

Figure 14: Domain for the battery problem.

\begin{tabular}{|c|c|}
\hline$x$ & $y$ \\
\hline \hline 0.0 & 0.0 \\
6.1 & 0.8 \\
6.5 & 1.6 \\
8.0 & 3.6 \\
8.4 & 18.8 \\
& 21.2 \\
& 23.2 \\
& 24.0 \\
\hline
\end{tabular}

Table 3: The locations of the line segments that separate the regions of the battery problem. For example, the line $y=24.0$ is the top of the region in Figure 14

\begin{tabular}{|c|c|c|}
\hline side & $c$ & $g_{N}$ \\
\hline \hline left & 0.0 & 0.0 \\
top & 1.0 & 3.0 \\
right & 2.0 & 2.0 \\
bottom & 3.0 & 1.0 \\
\hline
\end{tabular}

Table 4: Boundary condition coefficients for the four sides of the battery problem domain in Figure 14 

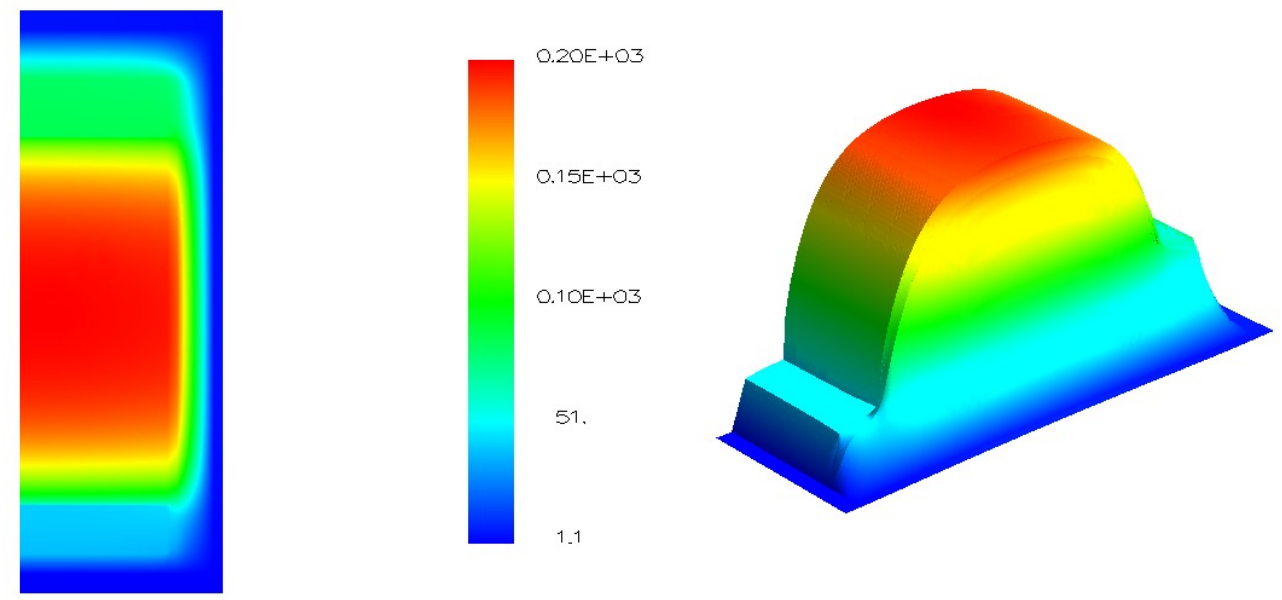

Figure 15: The solution of the battery problem.
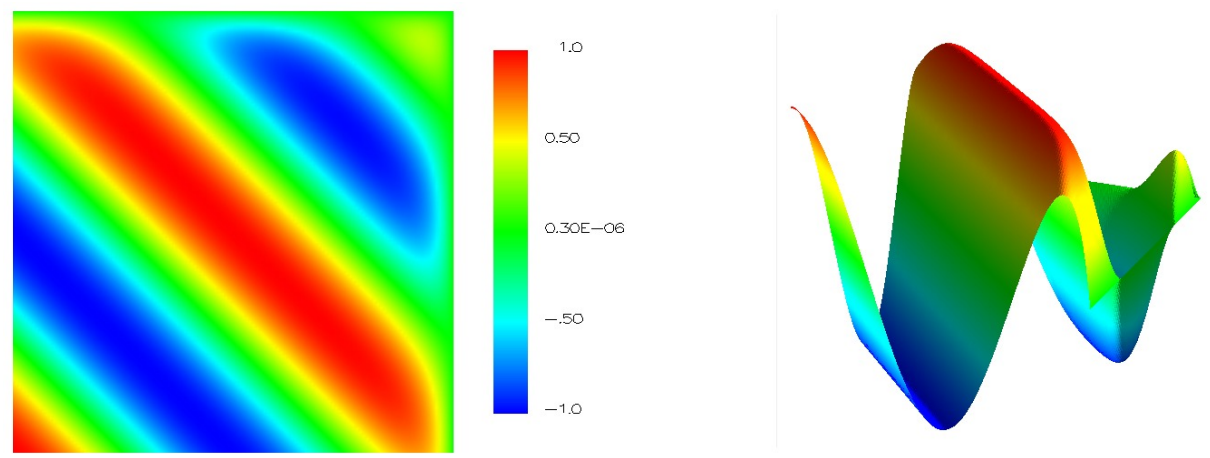

Figure 16: The solution of the boundary layer problem with $\epsilon=10^{-1}$.

Equation: $-\epsilon \nabla^{2} u+2 \frac{\partial u}{\partial x}+\frac{\partial u}{\partial y}=f$

Domain: $(-1,1) \times(-1,1)$

Boundary conditions: Dirichlet

Solution: $\left(1-e^{-(1-x) / \epsilon}\right)\left(1-e^{-(1-y) / \epsilon}\right) \cos (\pi(x+y))$

Parameters: $\epsilon$ determines the strength of the boundary layer.

Figures 16 and 17 show solutions with a mild boundary layer from $\epsilon=10^{-1}$, and a steep boundary layer from $\epsilon=10^{-3}$.

\subsection{Boundary Line Singularity}

Many papers [6, 9, 10, 11] use a 1D example with a singularity of the form $x^{\alpha}$ at the left endpoint of the domain. The solution is in $H^{\alpha+1 / 2-\epsilon} \forall \epsilon>0$ [9]. This can be extended to $2 \mathrm{D}$ by simply making the solution be constant in $y$. On the unit square, the result is a solution that is singular along the left boundary. 

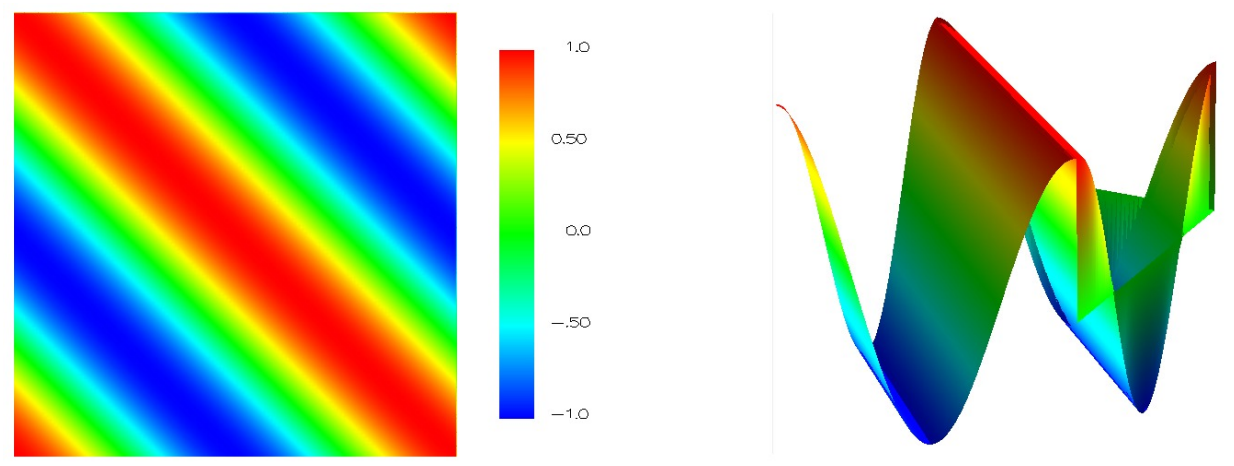

Figure 17: The solution of the boundary layer problem with $\epsilon=10^{-3}$.
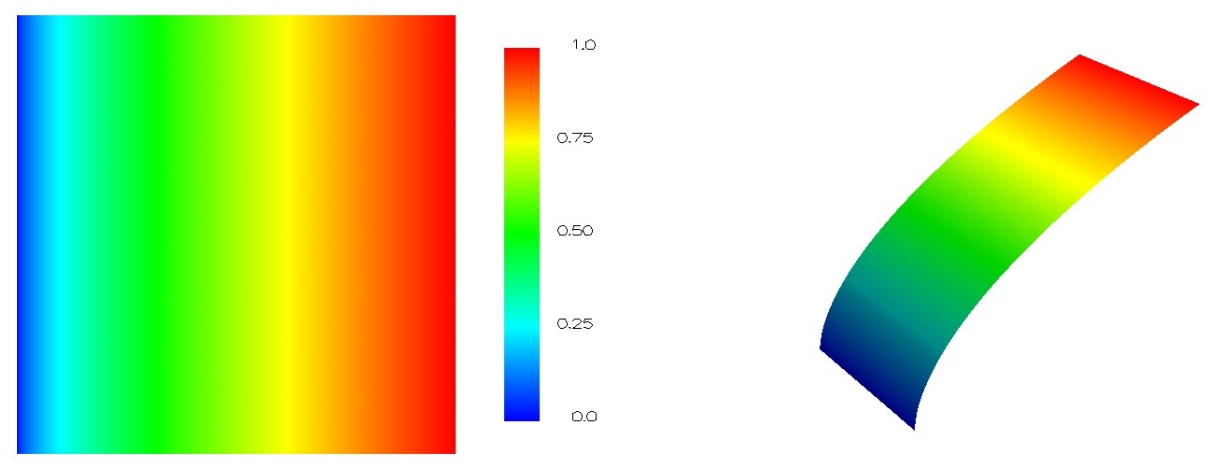

Figure 18: The solution of the boundary line singularity problem with $\alpha=0.6$.

Equation: Poisson

Domain: Unit square

Boundary conditions: Dirichlet

Solution: $x^{\alpha}$

Parameters: $\alpha \geq 1 / 2$ determines the strength of the singularity. All of the cited references use $\alpha=0.6$.

The solution with $\alpha=0.6$ is shown in Figure 18 .

\subsection{Oscillatory}

This problem is inspired by the wave function that satisfies a Schrödinger equation model of two interacting atoms [12. It is highly oscillatory near the origin, with the wavelength decreasing closer to the origin. The number of oscillations, $N$, is determined by the parameter $\alpha=\frac{1}{N \pi}$. We use a Helmholtz equation for this problem.

Equation: $-\nabla^{2} u-\frac{1}{(\alpha+r)^{4}} u=f$, where $r=\sqrt{x^{2}+y^{2}}$

Domain: Unit square

Boundary conditions: Dirichlet 

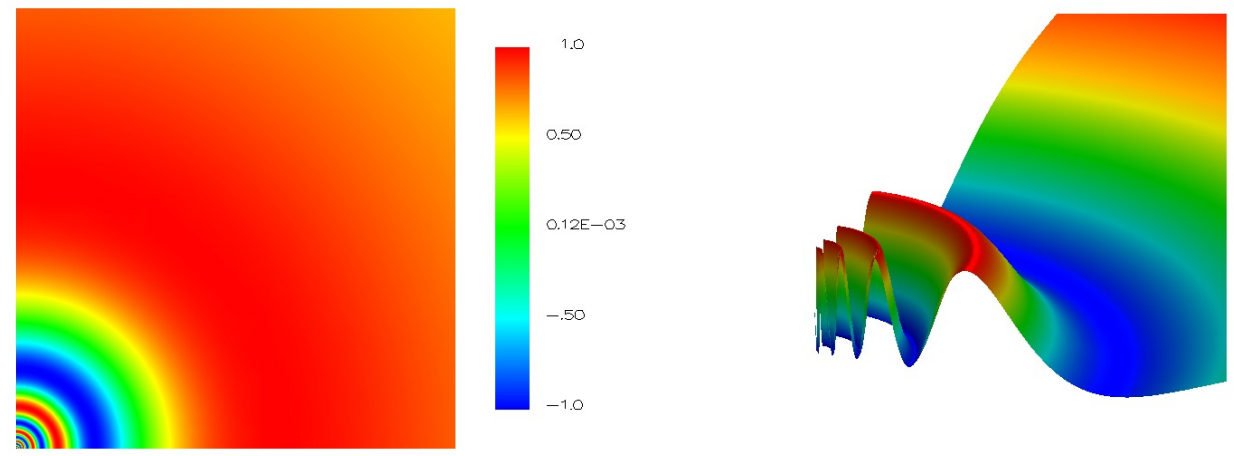

Figure 19: The solution of the oscillatory problem with $\alpha=\frac{1}{10 \pi}$
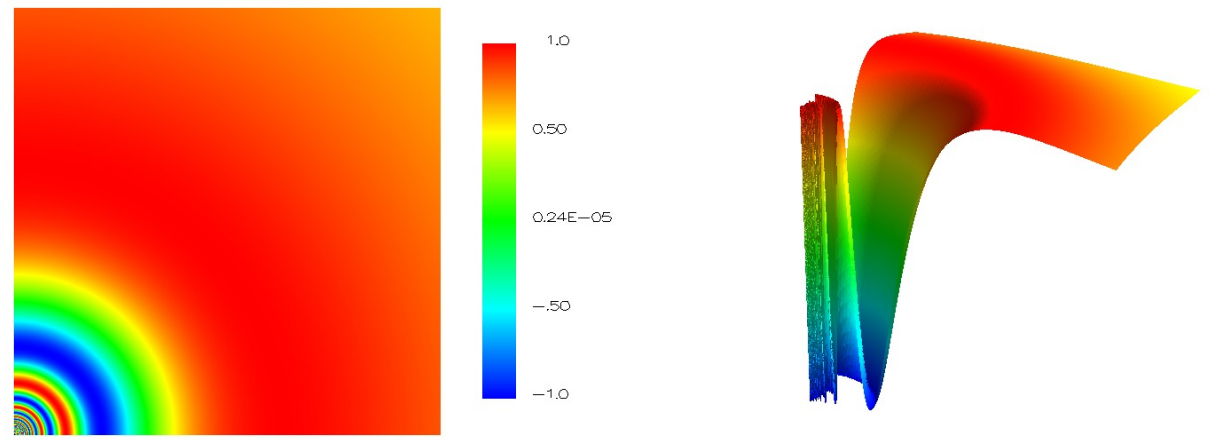

Figure 20: The solution of the oscillatory problem with $\alpha=\frac{1}{50 \pi}$

Solution: $\sin \left(\frac{1}{\alpha+r}\right)$

Parameters: $\alpha$ determines the number of oscillations.

The solutions of a relatively easy problem with $\alpha=\frac{1}{10 \pi}$ and a more difficult problem with $\alpha=\frac{1}{50 \pi}$ are shown in Figures 19 and 20. In the surface plot of Figure 19 we have zoomed in on the origin to show the detail.

\subsection{Wave Front}

A commonly used example for testing adaptive refinement algorithms is Poisson's equation with a solution that has a steep wave front in the interior of the domain [2, 6. 9, 11. Usually the wave front is given by an arctangent, but sometimes a hyperbolic tangent is used. In this problem we use a circular wave front as in [6, 11]. Parameters determine the steepness and location of the wave front. With the arctangent wave front, there is a mild singularity at the center of the circle. By observing the convergence rate with uniform $h$-refinement, we estimate that the solution is in $H^{m}$ with $m \approx 2$ if the center of the circle is in the closure of the domain, and smooth otherwise. 


\begin{tabular}{|l|r|r|r|r|}
\hline name & $\alpha$ & $x_{c}$ & $y_{c}$ & $r_{0}$ \\
\hline \hline mild & 20 & -.05 & -.05 & 0.7 \\
steep & 1000 & -.05 & -.05 & 0.7 \\
asymmetric & 1000 & 1.5 & 0.25 & 0.92 \\
well & 50 & 0.5 & 0.5 & 0.25 \\
\hline
\end{tabular}

Table 5: Parameters for the wave front problem.
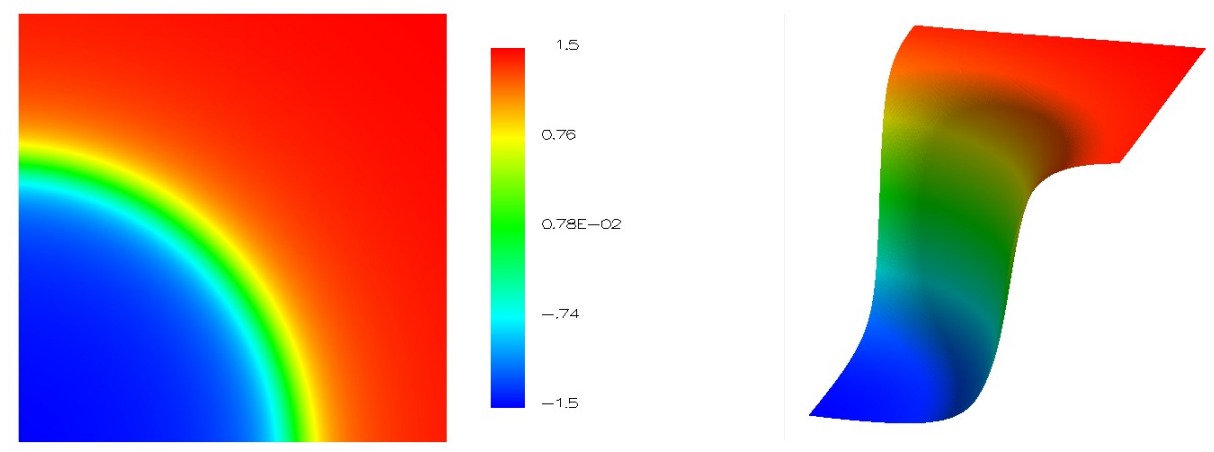

Figure 21: The solution of the mild wave front problem.

Equation: Poisson

Domain: Unit square

Boundary conditions: Dirichlet

Solution: $\tan ^{-1}\left(\alpha\left(r-r_{0}\right)\right)$ where $r=\sqrt{\left(x-x_{c}\right)^{2}+\left(y-y_{c}\right)^{2}}$

Parameters: $\left(x_{c}, y_{c}\right)$ is the center of the circular wave front, $r_{0}$ is the distance from the wave front to the center of the circle, and $\alpha$ gives the steepness of the wave front.

Four example solutions are shown in Figures 21, 24, with the parameters given in Table 5. In the first three we choose the center of the circle to be outside the domain so that we are examining the performance on the wave front, not the singularity. These solutions are characterized as a mild wave front, a steep wave front, and a steep wave front that is not symmetric about the origin. In the fourth example, the entire circle is inside the domain, resulting in a solution that is a well with a mild singularity at the bottom.

\subsection{Interior Line Singularity}

Houston et al. 9 extend the 1D $x^{\alpha}$ problem in Section 2.7 to $2 \mathrm{D}$ by extending the $1 \mathrm{D}$ domain to $(-1,1)$, defining the solution to be 0 for $x<0$, extending the domain to 2D with $y \in(-1,1)$, and adding $\cos (\pi y / 2)$. We extend this further to allow a sloped line so that the singularity does not necessarily coincide with element edges. There is no PDE in [9] since they are only evaluating their estimate of the regularity. We use a Poisson equation with Dirichlet boundary conditions. The solution is in $H^{\alpha+1 / 2-\epsilon} \forall \epsilon>0[9]$.

Equation: Poisson

Domain: $(-1,1) \times(-1,1)$ 

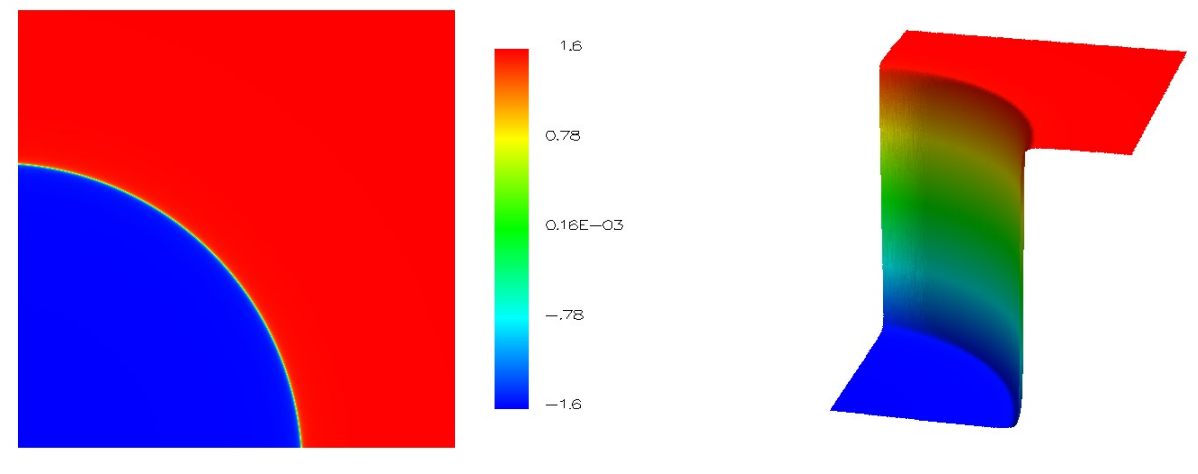

Figure 22: The solution of the steep wave front problem.
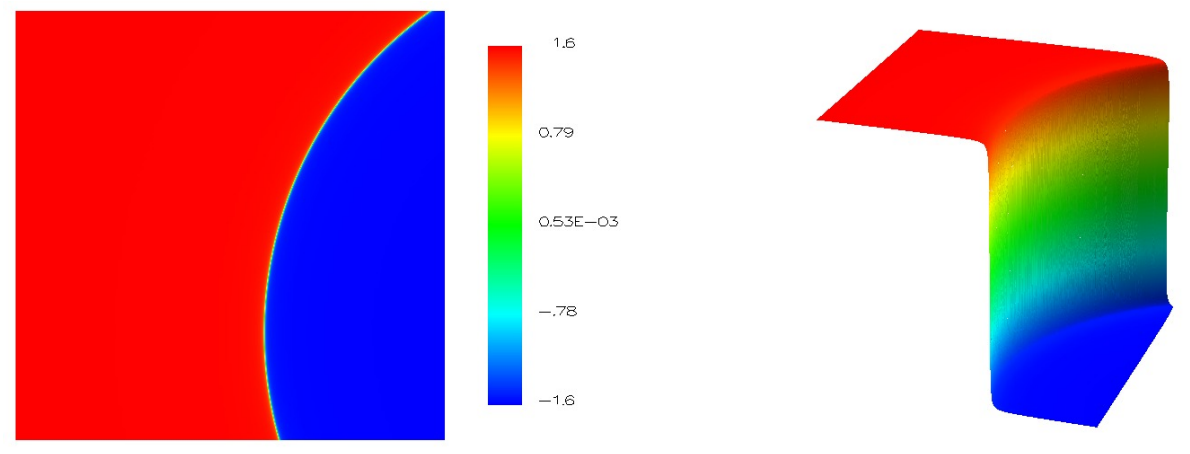

Figure 23: The solution of the asymmetric wave front problem.
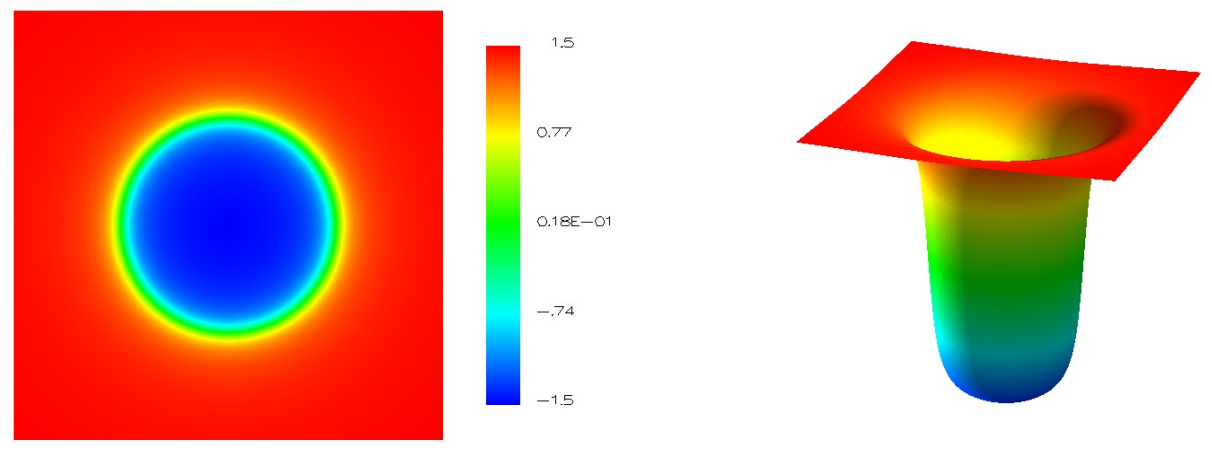

Figure 24: The solution of the wave front "well" problem. 

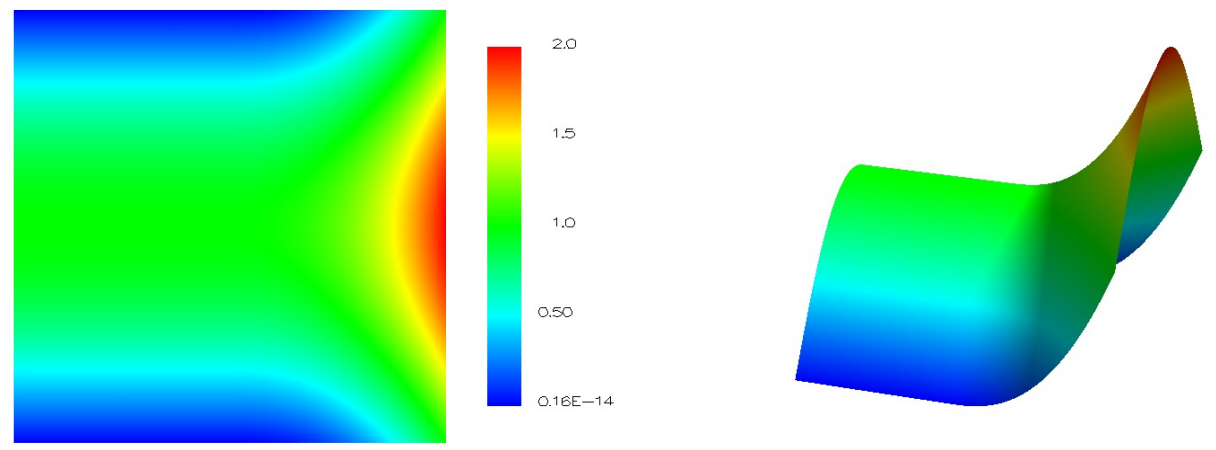

Figure 25: The solution of the interior line singularity problem with $\alpha=2.5$ and $\beta=0$.
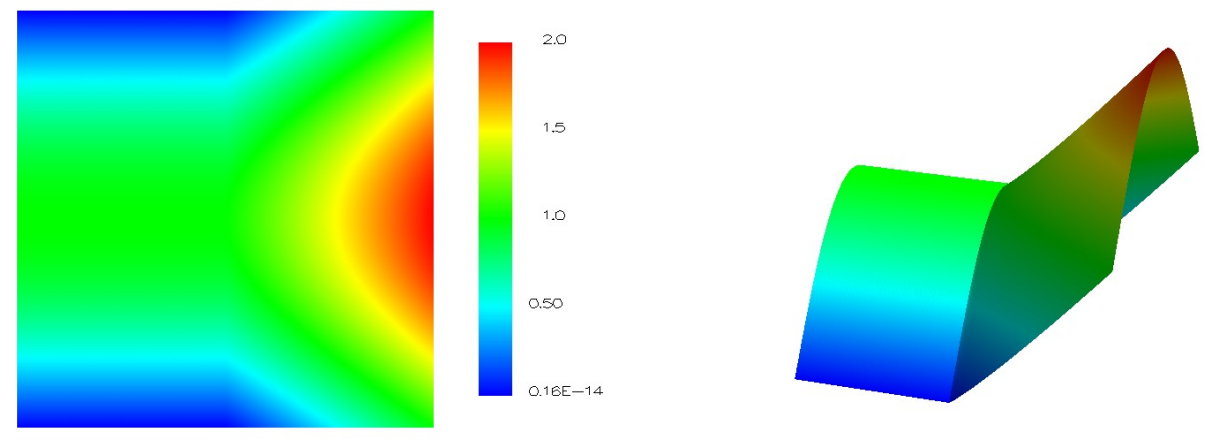

Figure 26: The solution of the interior line singularity problem with $\alpha=1.1$ and $\beta=0$.

Boundary conditions: Dirichlet

Solution: $\begin{cases}\cos (\pi y / 2) & x \leq \beta(y+1) \\ \cos (\pi y / 2)+(x-\beta(y+1))^{\alpha} & x>\beta(y+1)\end{cases}$

Parameters: $\alpha$ determines the strength of the singularity and $\beta$ determines the slope of the singularity line.

Solutions containing a mild singularity with $\alpha=2.5, \beta=0$, a stronger singularity with $\alpha=1.1, \beta=0$, and a slanted line singularity with $\alpha=1.5, \beta=0.6$ are shown in Figures 25.27.

\subsection{Intersecting Interfaces}

This problem comes from a paper by Kellogg 13 in which he studies Poisson problems with intersecting interfaces. Two interfaces, given by the lines $y=0$ and $y=\tan (\phi) x$, divide the plane into four regions for a given $\phi \in(0, \pi / 2]$. The PDE coefficients $p$ and $q$ are a piecewise constant function taking the value $p_{i}$ in the $i^{\text {th }}$ region counterclockwise from the positive $x$-axis. Let $\psi=\pi / 2-\phi$. The solution is given in polar coordinates by

$$
u=r^{a_{1}} \mu(\theta)
$$



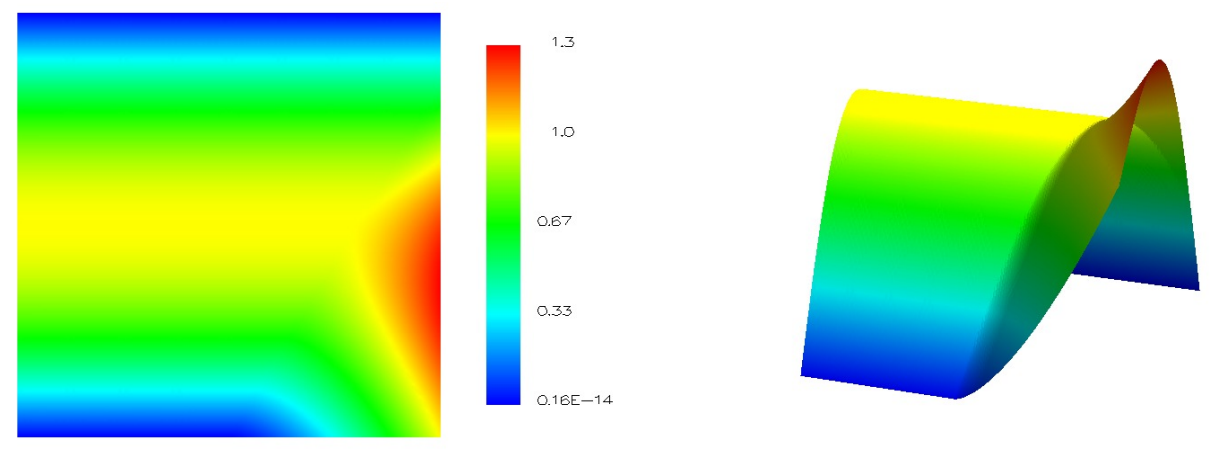

Figure 27: The solution of the interior line singularity problem with $\alpha=1.5$ and $\beta=0.6$.

where

$$
\mu(\theta)= \begin{cases}\cos \left(\left(\psi-\beta_{1}\right) a_{1}\right) \cos \left(\left(\theta-\phi+\alpha_{1}\right) a_{1}\right) & 0 \leq \theta \leq \phi \\ \cos \left(\alpha_{1} a_{1}\right) \cos \left(\left(\theta-\pi+\beta_{1}\right) a_{1}\right) & \phi<=\theta<=\pi \\ \left.\cos \left(\beta_{1} a_{1}\right) \cos \left(\theta-\pi-\alpha_{1}\right) a_{1}\right) & \pi<=\theta<=\pi+\phi \\ \cos \left(\left(\phi-\alpha_{1}\right) a_{1} \cos \left(\theta-\phi-\pi-\beta_{1}\right) a_{1}\right) & \pi+\phi<=\theta<=2 \pi\end{cases}
$$

and where the numbers $a_{1}, \alpha_{1}$ and $\beta_{1}$ satisfy the relations

$$
\left\{\begin{array}{l}
p_{1} / p_{2}=-\tan \left(\left(\psi-\beta_{1}\right) a_{1}\right) \cot \left(\alpha_{1} a_{1}\right) \\
p_{2} / p_{3}=-\tan \left(\alpha_{1} a_{1}\right) \cot \left(\beta_{1} a_{1}\right) \\
p_{3} / p_{4}=-\tan \left(\beta_{1} a_{1}\right) \cot \left(\left(\phi-\alpha_{1}\right) a_{1}\right) \\
p_{4} / p_{3}=-\tan \left(\left(\phi-\alpha_{1}\right) a_{1}\right) \cot \left(\left(\psi-\beta_{1}\right) a_{1}\right) \\
0<a_{1}<\pi / \psi \\
\max \left\{0,2 \phi a_{1}-\pi\right\}<2 a_{1} \alpha_{1}<\min \left\{2 \phi a_{1}, \pi\right\} \\
\max \left\{0, \pi-2 \psi a_{1}\right\}<-2 a_{1} \beta_{1}<\min \left\{\pi, 2 \pi-2 \psi a_{1}\right\}
\end{array}\right.
$$

The solution has a discontinuous derivative along the interfaces, and an infinite derivative at the origin. The solution is in $H^{1+a_{1}-\epsilon} \forall \epsilon>0$, so $a_{1}$ can be chosen to make the Sobolev regularity arbitrarily close to 1 .

Equation: $-\nabla \cdot p \nabla u=0$ where $p$ is piecewise constant

Domain: $(-1,1) \times(-1,1)$

Boundary conditions: Dirichlet

Solution: given by Equations 7 and 8

Parameters: As in [14, we take $\theta=\pi / 2, p_{1}=p_{3}=R, p_{2}=p_{4}=1$, and $a_{1}=0.1$. The conditions in Equation 9 can then be solved to obtain $R=161.4476387975881$, $\alpha_{1}=\pi / 4$ and $\beta_{1}=-14.92256510455152$.

The solution for the given parameters is shown in Figure 28.

\subsection{Multiple Difficulties}

In 11] one of the test cases involves both a singularity due to a reentrant corner and a sharp gradient in the form of an arctangent wave front. In this problem we combine 

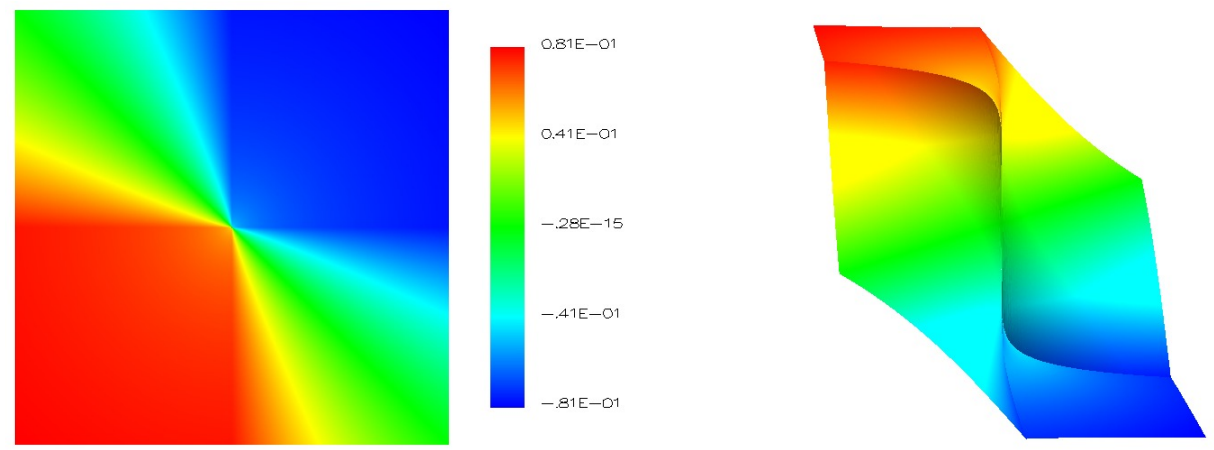

Figure 28: The solution of the intersecting interfaces problem.
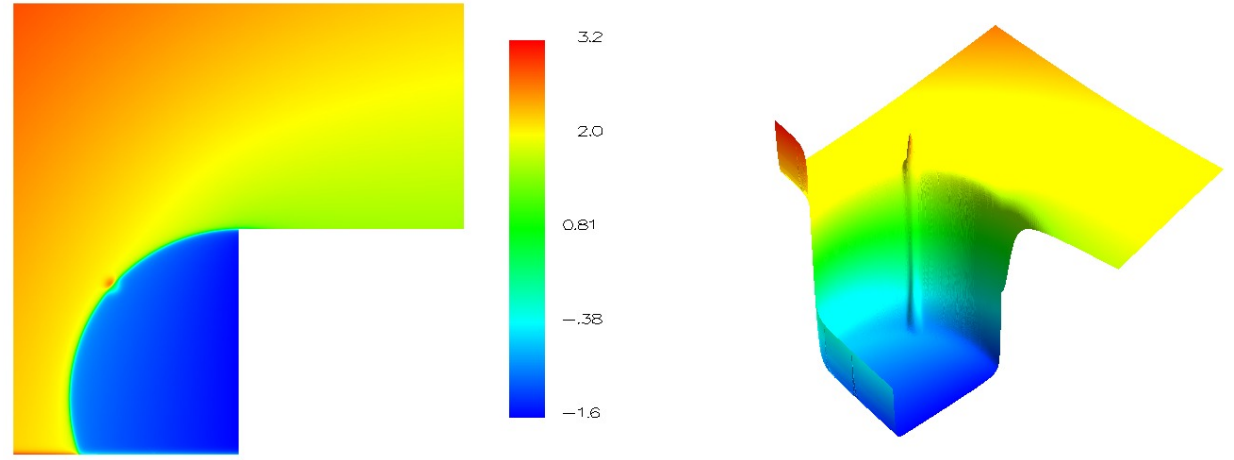

Figure 29: The solution of the problem with multiple difficulties.

four or five difficulties of different strengths into the same problem by combining some of the features of the other test problems. It contains a point singularity due to a reentrant corner, a circular wave front (which might include a singularity at the center of the circle), a sharp peak, and a boundary layer.

Equation: Poisson

Domain: L-shaped domain $(-1,1) \times(-1,1) \backslash(0,1) \times(-1,0)$

Boundary conditions: Dirichlet

Solution: $r^{(\pi / \omega)} \sin (\theta \pi / \omega)+\tan ^{-1}\left(\alpha_{w}\left(\sqrt{\left(x-x_{w}\right)^{2}+\left(y-y_{w}\right)^{2}}-r_{0}\right)\right)+$ $e^{-\alpha_{p}\left((x-x p)^{2}+\left(y-y_{p}\right)^{2}\right)}+e^{-(1+y) / \epsilon}$

Parameters: This problem has the same parameters as the problems that it combines. The boundary layer was placed on $y=-1$. It could instead be placed on $y=1, x=-1$ or $x=1$, or any combination of them.

Figure 29 shows a solution where the wave front intersects the boundary layer and corner singularity, and the peak is centered on the wave front. The following parameters were used. For the reentrant corner, $\omega=3 \pi / 2$. The wave front is defined by $\left(x_{w}, y_{w}\right)=$ $(0,-3 / 4), r_{0}=3 / 4$ and $\alpha_{w}=200$. The peak is centered at $\left(x_{p}, y_{p}\right)=(\sqrt{5} / 4,-1 / 4)$ with 
strength $\alpha_{p}=1000$. The boundary layer is given by $\epsilon=1 / 100$.

\section{Future Work}

The collection of problems given in this paper were selected to represent a variety of solution features for which adaptive grid refinement is appropriate in the solution of elliptic partial differential equations in 2D. Clearly this is a limited class of problems. Standard benchmark problems are also needed for 1D, 3D, time dependent problems, problems that are motivated by real-world applications without manufactured solutions, goal-oriented adaptivity, etc. A web resource [1] is being developed, from which researchers can obtain standard adaptive grid refinement benchmark problems from a rich collection of problems representing many classes of partial differential equations.

\section{References}

[1] W. F. Mitchell, NIST Adaptive Mesh Refinement Benchmark Problems web page, http://math. nist.gov/amr_benchmark

[2] J. T. Oden, A. Patra, A parallel adaptive strategy for $h p$ finite element computations, Comput. Methods Appl. Mech. Engrg. 121 (1995) 449-470.

[3] M. Ainsworth, B. Senior, Aspects of an adaptive $h p$-finite element method: adaptive strategy, conforming approximation, and efficient solvers, Comput. Methods Appl. Mech. Engrg. 150 (1997) $65-87$.

[4] I. Babuška, M. Suri, The $h-p$ version of the finite element method with quasiuniform meshes, RAIRO Modél. Math. Anal. Numér. 21 (1987) 199-238.

[5] I. Babuška, M. Suri, The $p$ - and $h-p$ versions of the finite element method, an overview, Comput. Methods Appl. Mech. Engrg. 80 (1990) 5-26.

[6] L. Demkowicz, Computing with hp-adaptive finite elements, Volume 1, One and two dimensional elliptic and Maxwell problems, Chapman \& Hall/CRC, Boca Raton, FL, 2007.

[7] J. Rice, E. Houstis, W. Dyksen, A population of linear, second order, elliptic partial differential equations on rectangular domains, Math. Comp. 36 (1981) 475-484.

[8] S. Adjerid, M. Aiffa, J. Flaherty, Computational methods for singularly perturbed systems, in: J. Cronin, R. O'Malley (Eds.), Singular Perturbation Concepts of Differential Equations, AMS, Providence, 1998.

[9] P. Houston, B. Senior, E. Süli, Sobolev regularity estimation for $h p$-adaptive finite element methods, in: F. Brezzi, A. Buffa, S. Corsaro, A. Murli (Eds.), Numerical Mathematics and Advanced Appplications, Springer-Verlag, Berlin, 2003, pp. 619-644.

[10] A. A. Novotny, J. T. Pereira, E. A. Fancello, C. S. de Barcellos, A fast $h p$ adaptive finite element mesh design for 2D elliptic boundary value problems, Comput. Methods Appl. Mech. Engrg. 190 (2000) 133-148.

[11] W. Rachowicz, J. T. Oden, L. Demkowicz, Toward a universal h-p adaptive finite element strategy, Part 3. Design of h-p meshes, Comput. Methods Appl. Mech. Engrg. 77 (1989) 181-212.

[12] W. F. Mitchell, E. Tiesinga, Adaptive grid refinement for a model of two confined and interacting atoms, Appl. Numer. Math. 52 (2005) 235-250.

[13] R. Kellogg, On the poisson equation with intersecting interfaces, Appl. Anal. 4 (1975) 101-129.

[14] P. Morin, R. H. Nochetto, K. G. Siebert, Data oscillation and convergence of adaptive FEM, SIAM J. Numer. Anal. 38 (2000) 466-488. 